\title{
Electrokinetically Control Flow Mixing in Microfluidic Chip Utilizing Microelectrode Based on Indium Tin Oxide
}

\author{
Tianyuan Lv ${ }^{1, \mathrm{a}}$, Ning Yang ${ }^{1,2, \mathrm{~b}}$, Zhiyu Zuo ${ }^{1, \mathrm{c}}$, Jianjiang Guo ${ }^{2, \mathrm{~d}}$ and Hu Huang, \\ ${ }^{1}$ Institute of Agricultural Engineering, Jiangsu University, Zhenjiang, PR China \\ ${ }^{2}$ School of Electrical and Information Engineering, Jiangsu University, Zhenjiang, PR China \\ ${ }^{3}$ Department of Biomedical Engineering, University of Minnesota, Minneapolis, United States \\ a18796086895@163.com, byangning7410@163.com, ${ }^{\mathrm{c}}$ zuozy@ujs.edu.cn, ${ }^{\mathrm{d}}$ jjguo18@foxmail.com ${ }^{\mathrm{e}}$ huhuang:hwangtiger@gmail.com
}

\begin{abstract}
In order to blend the liquid in the microfluidic chip adequately, this paper presented a novel mixing scheme in which the species streams was mixed via the application of Lorenz chaotic system algorithm to three pair of parallel electrodes mounted on the surfaces of the mixing chamber to produces chaotic electric fields. To select the better microelectrode arrays, the paper also discussed the advantages of the indium tin oxide (ITO) materials and used ITO to design the microelectrode array. Finally, simulations of the mixing process under the control of the proposed chaotic electric fields are performed. Analysis result shows that the correlation coefficient between the largest Lyapunov exponents on Lorenz chaotic system and chaotic flow behavior is 0.72 which shows a better synchronism, and it also proved that ITO is a better material to fabricate the microelectrode arrays.
\end{abstract}

Keywords. Microfluidic chip, fluid chaotic mixing, chaotic electric fields, indium tin oxide.

\section{Introduction}

Efficient and rapid fluid mixing is a critical component in microfluidic applications because the mixing effect determines the speed and quality of chemical reaction. However, due to the small size of microfluidic devices, fluid flow is constrained to the low Reynolds number regime, and hence species mixing occurs primarily as a result of diffusion which always makes the required mixing length very long to ensure satisfactory mixing results. In consequence, a requirement exists for improved mixing schemes capable of accelerating the species mixing effect [1].

Kinds of micromixers called passive micromixers are used to generate chaotic advection effect by using specific channel geometry configurations to split or stretch the fluid. Some designs were investigated such as the 3D serpentine micromixer, the zigzag micromixer [1], the rapid three-dimensional passive rotation micromixer, and the staggered herringbone micromixer [2]. In contrast to passive micromixers, active micromixers use the external force to split or stretch the fluid. When the size of channel is reduced to the microscale, the ratio of surface to volume ratio becomes large [3]. Thus, forces such as the surface tension and electrokinetic force become the dominated force instead of the volume forces. This enables new techniques to develop active micromixers. Some external forces are due to ultrasonic vibration, magnetic force and electroosmosis [4]. Oddy et al. [5], developed an electrokinetic instability micromixer in which a rapid stirring effect was obtained via the application of external sinusoidally alternating electric fields. Biddiss et al. [6-7], presented a rapid mixer which mixes the fluids by controlling the distribution of the non-uniform zeta potential to generate vortices on the surface.

Chaos synchronization is an evaluation index to judge the effect of chaos control [8]. Therefore, the chaos synchronization also can be used to judge control effect of electric fields on microfluid in the microfluidic chip. This paper presents a novel microfluidic mixing scheme in which the species streams are mixed via the application of Lorenz chaotic system algorithm to three pairs of electrodes which is designed by ITO.

\section{Materials manufacturing methods and physical models}

The principle of ITO microelectrode array. The ITO films $\left(\operatorname{In}_{2} \mathrm{O}_{3}\right)$ is transparent in the visible range (400 800nm), while the transmittance is above $90 \%$ and the reflectance in infrared region is more than $85 \%$. Such a high visible light transmittance and low resistivity makes the ITO films become the typical electrical properties of transparent conductive. The electrical characteristics of ITO films are measured by the sheet resistance and the thickness of R. Sn and the formation of oxygen vacancy makes the carrier concentration of ITO films become very high $\left(\sim 10^{20} \mathrm{~cm}^{3}\right)$, while its resistivity is relatively low $\left(\sim 10^{4} \Omega / \mathrm{cm}\right)$. So it forms a highly degenerate $\mathrm{N}$ type semiconductor, which shows a kind of metal. For degenerate semiconductor, the carrier concentration basically remain static with the change of temperature, and 
the electrical properties of materials mainly depends on the migration rate. Therefore, the above characteristics show that the choice of ITO as the electrode material is more suitable than the metal electrode.

The manufacturing of ITO microelectrode array. Physical models Figure 1a illustrate the whole geometric configuration of the microfluidic chip. A Y-type channel is used to allow two different fluids to be injected from the two side inlets A, B and mixed in the main mixing chamber, which contains three pair of parallel electrodes mounted on the surfaces.

Microfluidic chip is produced by polydimethylsiloxane (PDMS) material. The indium tin oxide (ITO) conductive film is chosen as substrate. Ultrasonic cleaning is carried out by the deionized water of methylbenzene, acetone and alcohol. The water is baked in the temperature of $120^{\circ} \mathrm{C}$. The negative photoresist ITO array electrode pattern can be obtained by 4 steps: whirl coating, prebake, exposure and developing. And then, the microelectrode array $(20 \mu \mathrm{m} \times 20 \mu \mathrm{m})$ is fabricated by ways of hardbaking, corrosion, removing of photoresist, scouring, stoving and so on. Figure $1 \mathrm{~b}$ is the photo of microelectrode array. The overall microfluidic system can be obtained by the way of plasma bonding which is between ITO substrate with a microelectrode array and PDMS chip.

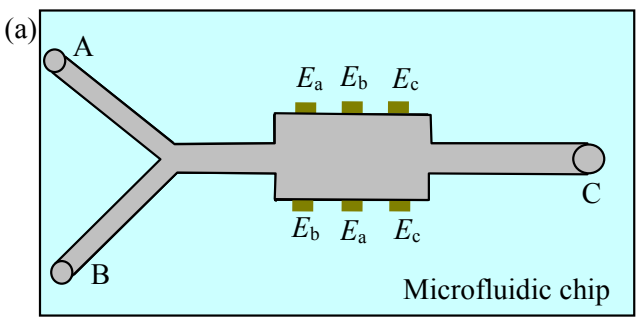

(b)

Fig. 1. The Y-shaped channel microfluidic chip chart.

(a) The whole geometric configuration of the microfluidic chip.

(b) The Photo of microelectrode array.

TablesChaotic system algorithm. The object of this study is the chaotic mixing effect of mircrofluid perturbed by application of chaotic electrical fields provided by chaotic system algorithm. Therefore, in the eyes of Chaos anti-control theory, chaotic system can be regarded as control system and microfluid system can be regarded as controlled system. The model can be used to describe the chaotic phenomenon of fluid. Accordingly, we chose Lorenz chaotic system as the control system shown in the follow equations:

$$
\frac{d x}{d t}=-c(x-y) ; \frac{d y}{d t}=a x-y ; \frac{d z}{d t}=x y-b z
$$

Where $x, y$ and $z$ are the oscillation signals applied to the three pair of parallel electrodes mounted on the surfaces of the mixing chamber; $b$ and $c$ are dimensionless parameters. $a$ is the characteristic parameter of the Lorenz chaotic system. To ensure the Lorenz chaotic system exhibits chaotic behavior, the initial values are set at $x(0)=y(0)=z(0)=1$, with the parametric values $\mathrm{c}=10, \mathrm{a}=28$ and $\mathrm{b}=8 / 3$. Under these settings, the signal $x,-y$ and $z$-a are applied to the electrodes $E_{\mathrm{a}}, E_{\mathrm{b}}$, and $E_{\mathrm{c}}$ which are shown in Figure 1a. The phase trajectory of the control variables $\mathrm{x},-\mathrm{y}$ and z-a varying with time is shown in Figure 2a. Figure $2 \mathrm{~b}$ shows the oscillatory trajectories of the control variables variables $\mathrm{x},-\mathrm{y}$ and $\mathrm{z}-\mathrm{a}$ varying with time. The electric potentials $\varphi_{\mathrm{E}}$ of the three pair of parallel electrodes are $\beta x,-\beta y$ and $\beta(z-\mathrm{a})$. Where $\beta$ is the amplification factor, In this study, the amplification factor $\beta=1.2$. Therefore, the oscillatory intensity $\beta x,-\beta y$ and $\beta(z-a)$ applied at electrodes $E_{\mathrm{a}}$, $E_{\mathrm{b}}$ and $E_{\mathrm{c}}$ ranges between -28.9 and $26.2 \mathrm{~V}$.

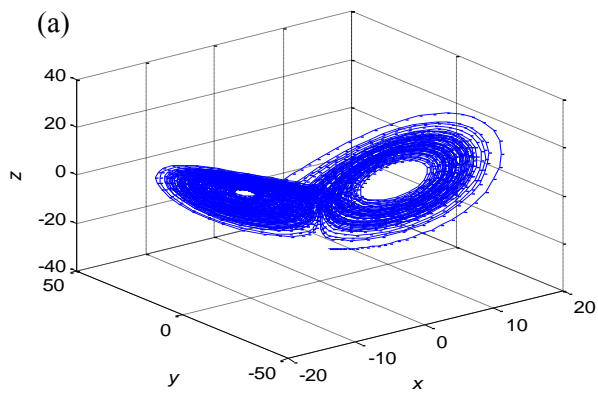

(b)

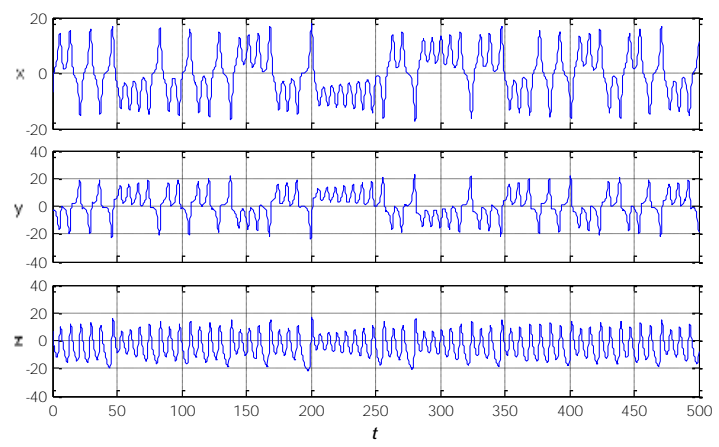

Fig. 2. Trajectories in the Lorenz system.

\section{Results and discussion}

COMSOL Multiphysics 4.3 was used to investigate the effect of the chaotic electric fields on the fluid flow characteristics within the mixing chamber. Figure 3 shows the initial distribution of the species concentration prior to the application of 
the chaotic electric fields. It is apparent that the two streams are essentially laminar flows which can be regarded as ordered microfluid system. Consequently, species mixing takes place primarily as a result of diffusion and initial mixing performance is poor.

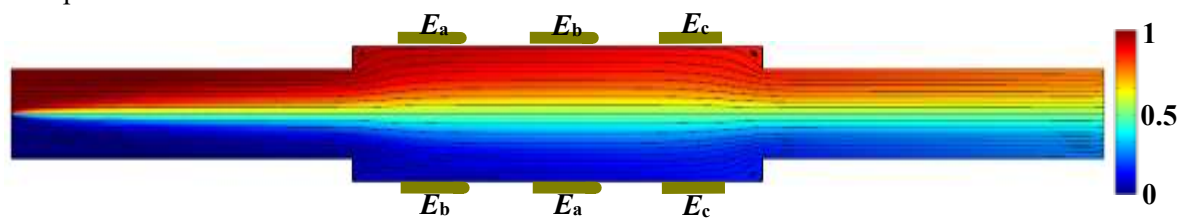

Fig. 3. The initial distribution of the species concentration prior to the application of the chaotic electric fields.

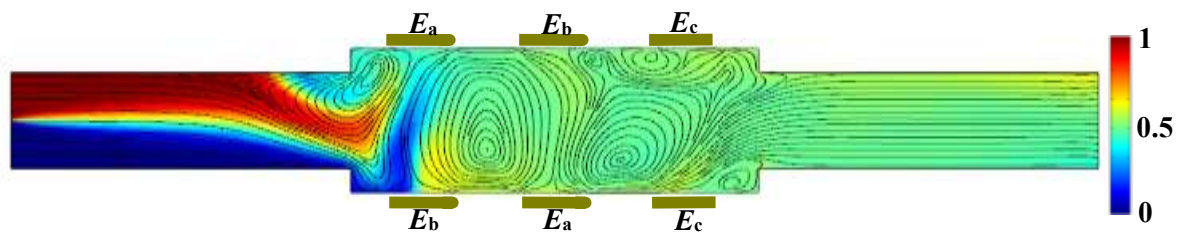

Fig. 4. The concentration and flow streamlines distribution within the mixing chamber with application of chaotic electrical field provided by Lorenz chaotic system at $\mathrm{a}=28$ and $\mathrm{t}=300 \mathrm{~ms}$.

Figure 4 illustrates the concentration and flow streamlines distribution within the mixing chamber with application of chaotic electrical field provided by Lorenz chaotic system at $a=28$ and $t=300 \mathrm{~ms}$. As shown, at this particular time instant, the electric potentials $\varphi_{E}$ at electrodes $E_{\mathrm{a}}, E_{\mathrm{b}}$ and $E_{\mathrm{c}}$ have a value of -15.2,27.3 and 6.8 respectively. These circulations full of the mixing chamber make the microfluid generate a chaotic mixing effect.

The chaotic evaluation of control system. Since the control system is the known Lorenz chaotic system, we obtained the accurate Lyapunov exponent spectrum by using numerical iteration method to compute the differential equation. On the whole, we solved the approximate solution of the ordinary differential equations firstly. Sencondly, orthogonalization and reiteration were carried out to obtain the Lyapunov exponent at each time point.

We adjust the characteristic parameter " $a$ " of the Lorenz chaotic system from 0 to 270 . Figure 5 shows the largest lyapunov exponents vary with the characteristic parameter. When $a<10$, the largest lyapunov exponent is a negativevalue, which indicates that the Lorenz system is cyclical change. While, when $a=32$, the largest lyapunov exponent reaches a maximum, which indicates that the intensity of chaos reaches the maximum value. While, as $a>200$, the largest lyapunov exponent reduces to 0 , which indicates that the Lorenz system return to cyclical change.

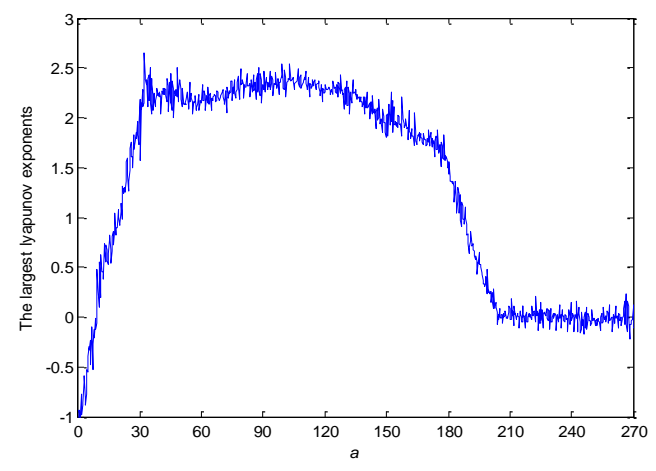

Fig. 5. The largest lyapunov exponents vary with the characteristic parameter.

The chaotic evaluation of controlled system. Because the controlled system is a microfluid system, the construction of the dynamic system by equation is difficult. Therefore, we have to reconstruct the series time of variables in gamma space and calculate the intensity of chaos by particle tracing method. The Particle Tracing Module in COMSOL Multiphysics version 4.3 provide us an environment for computing the trajectory of particles in the microfluidic mixer. Fig.6 shows the particle tracing at $300 \mathrm{~ms}$ in the mixing chamber. Wolf et al., present algorithms that allow the estimation of Lyapunov exponents from a time series of particle tracing. Based on the wolf method, we can judge the state of system by largest lyapunov exponent $\left(\lambda_{1}\right)$, second-largest lyapunov exponent $\left(\lambda_{2}\right)$ and third-largest lyapunov exponent $\left(\lambda_{3}\right)$. Though Wolf method provides the algorithms to estimate the Lyapunov exponents of system, the computation complexity is high and the result is susceptible to parameters. In fact, as long as the positive Lyapunov exponent exists, the microfuild system is a chaotic system this time. Therefore, there is no need to estimate the Lyapunov exponent spectrum from time series, just estimating largest lyapunov exponent is enough to judge the state of system. 


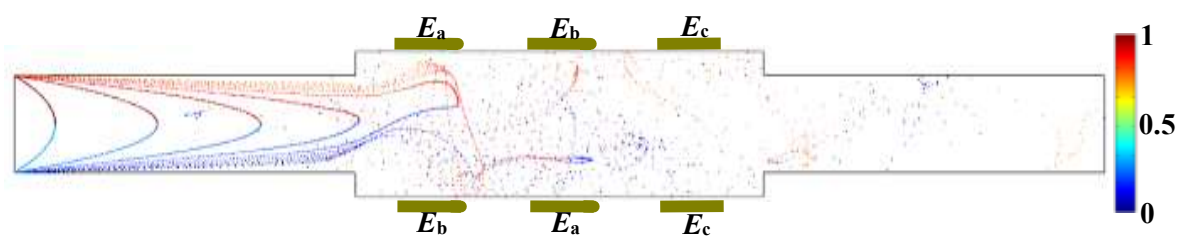

Fig. 6. The particle tracing at $300 \mathrm{~ms}$ in the mixing chamber.

Chaotic synchronization. From the variation of the largest Lyapunov exponents in Figure 5 and Figure 6 , It may be observed that the microfluidic system is synchronous with the Lorenz system. To figure this out, the relationship of the largest Lyapunov exponents between Lorenz system and microfluidic system are analyzed with regression analysis. Figure.7a shows the regression curve of the largest Lyapunov exponents between the system and controlled system. We commonly use the cross-correlation coefficient (also called the correlation coefficient) to describe the linear correlation between the data. $R$ represents the correlation coefficient. The relationship between $|R|$ and correlation degree are shown in table 1.

The correlation coefficient $R$ of the largest Lyapunov exponents between the two systems is 0.72 , which indicates the chaos intensity of the two systems have a good synchronization, and these results also prove that ITO is a better material to fabricate the microelectrode arrays. However, from Figure 5 and Figure $7 \mathrm{~b}$, we can discover that when the characteristic parameter $a$ of Lorenz system over 200, the largest Lyapunov exponents of Lorenz system fall to 0, while the largest Lyapunov exponents of microfluidic system is still greater than 0. Meantime, the Lorenz system is in a critical state, while the microfluidic system is in a chaotic state, though the intensity of chaos is small. The result indicates the state of the controlled system is not completely synchronous with the control system. However, When the characteristic parameter of chaotic electric fields is smaller than 200, the microfluidic system is well synchronous with the Lorenz system.

(a)

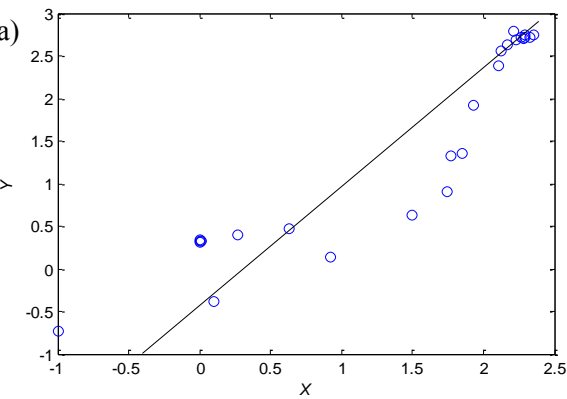

(b)

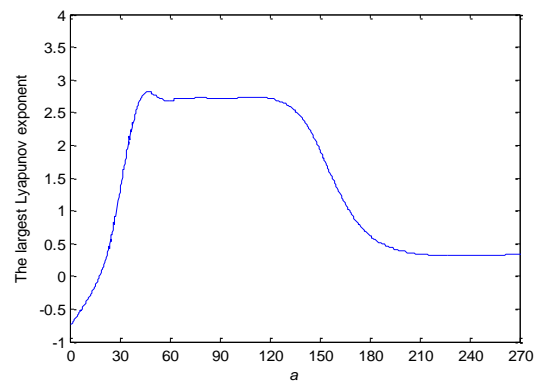

Fig.7. (a) The regression curve of the largest Lyapunov exponents between the system and controlled system. (b) The largest lyapunov exponents of the controlled microfluid system vary with characteristic parameter of the Lorenz chaotic control system.

Table 1. Relation between $|\mathrm{R}|$ and correlation degree.

\begin{tabular}{|c|c|c|c|c|c|}
\hline Range of $|R|$ & $0.00-0.19$ & $0.20-0.39$ & $0.40-0.69$ & $0.70-0.89$ & $0.90-1.00$ \\
\hline Correlation degree & Extreme low & Low & Moderate & High & Extreme high \\
\hline
\end{tabular}

\section{Summary}

This study has presented a novel microfluidic mixing scheme in which the species streams are mixed via the application of Lorenz chaotic system algorithm to three pair of parallel electrodes mounted on the surfaces of the mixing chamber to produces chaotic electric fields. And the microelectrode array is designed by the indium tin oxide (ITO) material. The advantages of ITO materials for the fabrication of microelectrode arrays are discussed. Simulations have been performed to investigate the control effect of the chaotic electric field on fluid chaotic characteristics. The simulation results have shown that the correlation coefficient between the largest Lyapunov exponents on Lorenz chaotic system and chaotic flow behavior is 0.72 which shows a better synchronism, and it also proved that ITO is a better material to fabricate the microelectrode arrays. When the characteristic parameter of Lorenz system is smaller than 200, the state of the chaotic flow system keep pace well with Lorenz system. This work is useful to the design of the mixing chamber via the application of chaotic electric fields to promote microfluid mixing in microfluidic chip. 


\section{Acknowledgements}

Supported by the Priority Academic Program Development of Jiangsu Higher Education Institutions(PAPD), Natural Science Foundation of Jiangsu Province(BK20140550), China Postdoctoral Science Foundation (2015T80512), Changzhou Sci\&Tech Program(CE20155054)and Research and Development Plan of Zhenjiang to Modern Agriculture(NY2015026).

\section{References}

1. S. J. Park, J. K. Ki2m, J. Park, S. Chung, C. Chung, K. Chang. Rapid three-dimensional passive rotation micromixer using the breakup process J, Micromech. Microeng, Vol. 12(2004), 6-14.

2. H. J. Song, Z. L. Cai, H. M. Noh, D. J. Bennett. Chaotic mixing in microchannels via low frequency switching transverse electroosmotic flow generated on integrated microelectrodes, Lab Chip, Vol. 10(2010), p. 734-740.

3. G. A. Mensing, T. M. Pearce, M. D. Graham, D. J. Beebe. An externally driven magnetic microstirrer, Phil. Trans. Roy. Soc, Vol. 362(2004), p. 1059-1068.

4. S. Qian, H. H. Bau. A chaotic electroosmotic stirrer. Anal, Chem. Vol. 74(2002), p. 3616-3625.

5. M. H. Oddy, J. G. Santiago, J. C. Mikkelsen. Electrokinetic instability micromixer, Anal, Chem., Vol. 73(2001), p.5822-5832.

6. E. Biddiss, D. Erickson, D. Li. Heterogeneous surface charge enhanced micromixing for electrokinetic flows, Anal. Chem,Vol. 76(2004), p.3208-3213.

7. H. J. Song, J. B. Dawn. Numerical study of enhancing the mixing effect in microchannels via transverse electroosmotic flow by placing electrodes on top and bottom of the channel, Microsyst. Technol, Vol. 17(2011), p.1427-1437.

8. S. Das, I. Pan, S. Das, A. Gupta. Master-slave chaos synchronization via optimal fractional order (PID mu)-D-lambda controller with bacterial foraging algorithm, Nonlinear Dynam, Vol. 69(2012), p.2193-2206. 